\title{
AN EXTENDED MATRIX EXPONENTIAL FORMULA
}

\author{
HEESEOP KIM AND YONGDO LIM
}

Abstract. In this paper we present matrix exponential formulae for the geometric and spectral geometric means of positive definite matrices using a conjectured exponential formula that solved by Wasin So [Linear Algebra Appl. 379 (2004)].

Mathematics subject classification (2000): 15A24, 15A29, 15A48.

Key words and phrases: Exponential formula, Hermitian matrix, positive definite matrix, geometric and spectral mean, Lie-Trotter formula.

\section{REFERENCES}

[1] E. Ahn, S. Kim AND Y. Lim, An extended Lie-Trotter formula and its applications, Linear Algebra Appl. (to appear)

[2] T. ANDO, Topics on Operator Inequalities, Lecture Notes Hokkaido Univ., Sapporo, 1978.

[3] R. BhatiA, Matrix Analysis, Graduate Texts in Mathematics, Springer, 1996.

[4] R. BHATIA, On the exponential metric increasing property, Linear Algebra Appl. 375 (2003), 211-220.

[5] M. FIEDLER AND V. PTÁK, A new positive definite geometric mean of two positive definite matrices, Linear Algebra Appl. 251 (1997), 1-20.

[6] S. GolDEN, Lower bounds for Helmholtz function, Phys. Rev. 137 (1965), 1127-1128.

[7] F. HiaI AND D. PETZ, The Golden-Thompson trace inequality is complemented, Linear Algebra Appl. 181 (1993), 153-185.

[8] S. LANG, Fundamentals of differential geometry, Graduate Texts in Mathematics 191, 1999.

[9] J. D. LAWSON AND Y. LIM, The geometric mean, matrices, metrics, and more, Amer. Math. Monthly 108 (2001), 797-812.

[10] W. So, The high road to an exponential formula, Linear Algebra Appl. 379 (2004), 69-75.

[11] W. SO AND R. C. THOMPSON, Product of exponentials of Hermitian and complex symmetric matrices, Linear and Multilinear Algebra 29 (1991), 225-233.

[12] C. J. Thompson, Inequalities and partial orders on matrix spaces, Indiana Univ. Math. J. 21 (1971), 469-480. 